\title{
Non Linear Electrodynamics Contributing to a Minimum Vacuum Energy ("Cosmological Constant") Allowed in Early Universe Cosmology
}

\author{
Andrew Walcott Beckwith \\ Physics Department, Chongqing University Huxi Campus, Chongqing, China \\ Email:Rwill9955b@gmail.com
}

Received 4 November 2015; accepted 28 December 2015; published 31 December 2015

Copyright (C) 2016 by author and Scientific Research Publishing Inc.

This work is licensed under the Creative Commons Attribution International License (CC BY). http://creativecommons.org/licenses/by/4.0/

\section{(c) (7) Open Access}

\section{Abstract}

This article poses the question of a minimum cosmological constant, i.e. vacuum energy at the start of the cosmological evolution from a near singularity. We pose this comparing formalism as given by Berry (1976) as to a small time length, and compare that in its entirety to compare this value given by Berry (1976) with a minimum time length at the start of cosmological space-time evolution. Using the methodology of Zeldovich (1972) as to a problem with electron-positron pair production we also propose another upper bound to the problem of minimum time length which may be accessible to experimental inquiry. This then makes the problem of minimum time length a way of specifying a magnetic field dependence of the cosmological constant, which has major implications to answering if quintessence, $i . e$. a changing cosmological vacuum energy, or a constant for the "cosmological constant" problem. Our answer is an initial value for the cosmological vacuum energy $10^{10}-1^{20}$ times greater than today which suggests either Quintessence, or if still a constant, a much better value for this parameter than what is suggested by traditional field theory methods. In closing we review how our construct supports work done by Corda, as to early universe models and what the implications are, as to the choices we have made.

\section{Keywords}

Cosmological Vacuum Energy, Energy Density, Initial Time Step

\section{Introduction}

We first of all cite that there exists formalism of Berry [1] as to the formation of a shortest time step consistent to FRW space-time metrics. Then we will compare the results to the derived minimum time step derivation 
which is consistent with Non linear Electrodynamics NLED. In doing so we will isolate what is a range of values for the vacuum energy, and "cosmological constant" dependent upon Nonlinear Electrodynamics NLED [2] inputs

Berry's results [1] are that for a standard flat space FRW cosmology, with positive cosmological "constant"

$$
t \sim \frac{2}{\sqrt{3 \Lambda}} \cdot \operatorname{arcsinh}\left[a(t) \cdot \sqrt{\frac{\Lambda}{8 \pi G \rho}}\right]
$$

We will compare this answer with a minimum time step consistent with Non linear Electrodynamics NLED and use it to obtain a Non linear Electrodynamics NLED bound on the $\Lambda$ cosmological constant term.

\section{First the Non Linear Electrodynamics NLED Inputs into a Minimum Time Step}

If we consider the role of an electromagnetic charge, as freely given in this presentation, we should also look at a derivation of what Zel'dovich [3] gave as far as charge and anti charge particles, in an applied E and M field could yield, which would be of the form (when $\xi$ is an energy expression, and $\mathrm{E}$ an applied electric field of the value of)

$$
\frac{\mathrm{d} \xi}{\mathrm{d} t}<\frac{e E \xi}{m c}
$$

Again to generalize this Equation (2), we consider if the electric field is such that the commensurate bulk charge will have the following relationship to the given electromagnetic charge, to read as, if when $\xi$ is an energy expression, with in Equation (3) below

$$
\begin{aligned}
& \mathrm{d} E=-4 \pi j \mathrm{~d} t \Rightarrow E=-4 \pi j t \\
& \& j=2 e n c \\
& \Rightarrow(\Delta t)^{2} e \cdot n \cdot c=\frac{m}{4 \pi c} \ln \frac{\xi_{\text {init }}}{\xi_{\text {final }}} . \\
& \Rightarrow e=\frac{m}{4 \pi n c^{2} \cdot(\Delta t)^{2}} \ln \frac{\xi_{\text {init }}}{\xi_{\text {final }}}
\end{aligned}
$$

Picking the time variation as given by $\Delta t$, the number of charges as given by $n$, and $m_{E \& M}$ the mass of a hypothetical "magnetic" charge as, derived by the author to read as

$$
e=\frac{m}{4 \pi n c^{2} \cdot(\Delta t)^{2}} \ln \frac{\xi_{\text {init }}}{\xi_{\text {final }}}=e_{E \& M} \sim \sqrt{\frac{B^{2} \cdot r_{\text {min }}}{\mu_{0} \cdot\left[1-2 \cdot \frac{B^{2} \cdot r_{\text {min }}}{c} \cdot X_{0}\left(\frac{B_{c}}{B}\right)\right]}}
$$

Equation (3) has a deeper meaning. That that not only is there a net "magnetic" monopole charge, as given in Equation (4), that there is a minimum non zero "energy density" of the electric and magnetic field (E and $\mathrm{M}$ ), as given by either $\xi_{\text {init }}$ or $\xi_{\text {final }}$ for an emergent "magnetic monopole" charge from an initial space-time configuration. This energy density value will lead to, to first order a minimum upper time step which we will characterize as [4]

$$
(\Delta t)^{2}=\frac{m}{4 \pi n c^{2} \cdot e} \ln \frac{\xi_{\text {init }}}{\xi_{\text {final }}}=\sim \sqrt{\frac{B^{2} \cdot r_{\min }}{\mu_{0} \cdot e^{2} \cdot\left[1-2 \cdot \frac{B^{2} \cdot r_{\min }}{c} \cdot X_{0}\left(\frac{B_{c}}{B}\right)\right]}}
$$

In doing so we will characterize the values to be set for Equation (4) as follows. i.e. $r_{\min }, \boldsymbol{n}, \boldsymbol{B}$, and energy densities as given by $\xi_{\text {init }}$ and $\xi_{\text {final }}$. These values come from the work of the author as interpreting [2] as well as applying the results for a minimum radial density which are implied by the minimum scale value for the initial "radii" of the universe due to electro dynamics given in [3], and of course the function $\mathrm{X}_{0}\left(\frac{B_{c}}{B}\right)$ given in [2]. 
The next section will give inputs into these values and will be used to show how non linear electrodynamics may -influence an upper bound choice for the minimum time step which may arise in the start of the evolution of the universe

\subsection{Examining Inputs for $r_{\min }, n, B$, and Energy Densities as given by $\xi_{\text {init }}$ and $\xi_{\text {final }}$}

In this section we will lay out arguments given in [2] to their logical conclusion as well as build upon several of the procedures given in [2] [3] to fill in these quantities, so as to give an upper bound to the minimum time step as referenced in Equation (5). To do so it would be useful to look at the formalism of Zeldovich [2] very closely, and if that is done, we can assert that the nucleation life-time for an electron-positron pair is give by setting $\Delta t \sim \tau_{e^{+}-e^{-} \text {pair }}$ with $\tau_{e^{+}-e^{-} \text {pair }}$ defined as below

$$
\tau_{e^{+}-e^{-} \text {pair }} \sim \frac{m_{e^{+}, e^{-C}}}{e E}
$$

Then the numerical density of the electron-positron pair may be given as

$$
n \sim \frac{e E^{2}}{4 \pi m c^{4}} \cdot \ln \left[\frac{\xi_{\text {initial }}}{\xi_{\text {final }}}\right]
$$

We will next begin to analyze what should be for $\xi_{\text {init }}$ and $\xi_{\text {final }}$. Which will in turn lead to the 'net' electric field, i.e. the net " $\mathrm{E}$ and $\mathrm{M}$ " field is, in reality a consequence of work in [2]-[4] which gives a minimum figure as to non linear electrodynamics and its consequence to a non zero initial radii of the universe. We go into this detail after giving order of magnitude estimates as to a minimum radii for the start of inflationary expansion.

\subsubsection{What Are Working Values for $\xi_{\text {init }}$ and $\xi_{\text {final }}$ as Well as Equation (6) $n$ Value?}

To engage on this, we use the Zeldovich [2] value for the net frequency which is given as

$$
\omega \sim m c^{2} / \hbar
$$

We assume that the net frequency remains in Initial nucleation invariant, but that the initial and final volumes change, by an amount we will quantify next, i.e. start with an initial to final radii of

$$
\begin{aligned}
& r_{\text {initial }} \sim 7.7 \times 10^{-30} \text { meters } \\
& r_{\text {final }} \sim 7.7 \times 10^{-29} \text { meters }
\end{aligned}
$$

Then

$$
\ln \left[\frac{\xi_{\text {initial }}}{\xi_{\text {final }}}\right] \sim 6.908
$$

This is the simplest interpretation of the consequence of varying energy density. Now for background to confirm it:

Making use of [2] [3], we assert that the minimum radii of Equation (8) is consistent with predictions of [3] [4], i.e. we are looking at what happens if an electron moves at a velocity of $v$, with

$$
|v|=|E / B|
$$

Implying if $\mathrm{c}$ is the speed of light, and $\tilde{\beta}>0$, then the magnitude of the electric field should be given by

$$
|E|=c \cdot 10^{-\tilde{\beta}}|B|
$$

i.e. in [2] we look at a generalized density.

$$
\rho=\frac{1}{2 \mu_{0}} \cdot B^{2} \cdot\left(1-8 \cdot \mu_{0} \cdot \omega \cdot B^{2}\right)
$$

This has a positive value only if input ( $E$ and $M$ ?) frequency $\omega$ is such that. 


$$
B<\frac{1}{2 \cdot \sqrt{2 \mu_{0} \cdot \omega}}
$$

In this situation we will be setting $B \equiv B_{0}$. What we are asserting is, that the very process of an existent $E$ and $\mathrm{M}$ field, also, sets a non zero initial radii to the universe, i.e. in [3] there exists a scaled parameter $\lambda$, and a parameter $a_{0}$ which is paired with

$$
\begin{aligned}
& \alpha_{0}=\sqrt{\frac{4 \pi G}{3 \mu_{0} c}} B_{0} \\
& \lambda=\Lambda c^{2} / 3
\end{aligned}
$$

Then if, initially, Equation (15) is large, due to a very large initial vacuum energy parameter $\Lambda$ the time, given in Equation (53) of [2] is such that we can write, most likely, that whenever one sees the coefficient like the magnetic field, with the small 0 coefficient, for large values of $\Lambda$, this should be the initial coefficient at the beginning of space-time which helps us make sense of the non zero but tiny minimum scale factor

$$
a_{\min }=a_{0} \cdot\left[\frac{\alpha_{0}}{2 \lambda}\left(\sqrt{\alpha_{0}^{2}+32 \lambda \mu_{0} \omega B_{0}^{2}}-\alpha_{0}\right)\right]^{1 / 4}
$$

The above scale factor should be such that the value of Eq. (17) should be in its smallest, i.e. 480,000 times proportionately larger than a Planck length of $l_{\text {Planck }} \sim 1.6162 \times 10^{-35}$ meters, i.e. scale $a_{0} \propto 10^{-29}$

Then, we will have that

$$
\begin{aligned}
& {\left[\frac{\alpha_{0}}{2 \lambda}\left(\sqrt{\alpha_{0}^{2}+32 \lambda \mu_{0} \omega B_{0}^{2}}-\alpha_{0}\right)\right] \geq 1} \\
& \Rightarrow \frac{\alpha_{0}^{2}}{2 \lambda}\left(\sqrt{1+32 \frac{\lambda \mu_{0} \omega B_{0}^{2}}{\alpha_{0}^{2}}}-1\right) \geq 1 \\
& \Rightarrow 8 \mu_{0} \omega B_{0}^{2} \geq 1
\end{aligned}
$$

Equation (18) puts a strong constraint upon the frequency and magnetic field strength, whereas Equation (12) gives a strong set of values as to allowed E, so as to have, then

$$
E^{2} \geq c^{2} \cdot 10^{-2 \tilde{\beta}} / 8 \mu_{0} \omega
$$

This value for the square of the electric field should be then put into obtaining

$$
n \sim \frac{e E^{2}}{2 m c^{4}} \sim \frac{10^{-2 \tilde{\beta}} \cdot e}{16 \mu_{0} \omega \cdot m c^{2}}
$$

The numerical "density" of electron-positron pairs drops as when the frequency $\omega$ rises. Which is counter intuitive, but it meshes well with

$$
\begin{aligned}
& (\Delta t)^{2} \sim \frac{m_{e}}{2 n c^{2} \cdot e} \\
& \underset{\omega \rightarrow \text { small }}{\longrightarrow} t_{\text {Planck }}^{2} \propto\left(5.39 \times 10^{-44}\right)^{2} \mathrm{sec}^{2} \\
& \underset{\omega \rightarrow \text { much-larger }}{\longrightarrow} \# \cdot t_{\text {Planck }}^{2}>\left(5.39 \times 10^{-44}\right)^{2} \mathrm{sec}^{2}
\end{aligned}
$$

i.e. for low frequency, we have a collapse to the Planck time frequency value, whereas, the minimum time step rises as frequency $\omega$ rises. Furthermore keep in mind that this result holds even if Equation (23) is formed in a way independent of a changing vacuum energy, as given by [2]

$$
\Lambda(t) \sim\left(H_{\text {inflation }}\right)^{2}
$$


Whereas this may tie into a massive graviton mass as given by the author as spin off of massive gravitons given in [5]

$$
m_{g}^{2}=\frac{\tilde{\kappa} \cdot \Lambda_{\max } \cdot c^{4}}{48 \cdot h \cdot \pi \cdot G}
$$

i.e. the time step is then independent upon elementary arguments as to massive graviton mass. Furthermore, even if [6]

$$
\Lambda_{0, M}=\frac{G}{32 \pi} \cdot m_{0}^{4} \cdot \exp \left[3 \cdot\left(\frac{m_{g}}{m_{0}}\right)^{4}\right]
$$

And there is a relationship between Equation (24) and Equation (22), as well as a density functional which may relate to initially scaled mass $m_{0}$, that due to a very weak linkage between a density functional and $m_{0}$, and Equation (20) that there is no clear linkage between Equation (21) and Equation (20), which is also counter intuitive We will next then compare Equation (21) with Berry's formalism [1] based on the FRW metric

\section{Minimum Time According to the FRW Metric Allows for a NLED Bound to $\Lambda$}

The easiest case to consider is, if the $\Lambda$ is not overly large, and the initial scale factor $a(t)$ is small. Then we have

$$
t \sim \frac{2}{\sqrt{3 \Lambda}} \cdot\left(a(t) \cdot \sqrt{\frac{\Lambda}{8 \pi G \rho}}-\frac{a^{3}(t)}{2.3}\left(\frac{\Lambda}{8 \pi G \rho}\right)^{3 / 2}+H O T\right)
$$

Then we are looking at

$$
\Lambda \sim \frac{8 \pi G \rho_{\text {galaxies }}}{a^{2}(t)} \cdot\left[1-\sqrt{\frac{3}{4}} \frac{m^{2} \mu_{0} \omega}{e^{2} a(t)} \sqrt{8 \pi G \rho_{\text {galaxies }}} \cdot 10^{2 \tilde{\beta}}\right]
$$

Here, $a\left(t_{\text {initial }}\right) \sim 10^{-30}$ is very small, but we are also assuming an ultra low $\rho_{\text {galaxies }}$ and $\omega$, and small $m$. The net effect is for a small positive $\Lambda$ as one is observing. This is different from the work done with String theory, as given in [7] below, which will be brought up in the conclusion.

$$
\frac{8 \pi G \rho_{\text {galaxies }}}{a^{2}(t)}<1
$$

We will next then discuss how our work is connected to what Corda has brought up in work which is to discriminate between different cosmological models. This section is copied from the paper to be published [8] by the author and is reproduced here, due to its relevance.

\section{Re-Examining Relic Gravitational Wave Models as to What Relic Gravitational Waves Could Tell Us about the Origins of the Early Universe. As Given in an Earlier Paper by the Author}

Quoting from [8] we write the following. We reproduce this, because of the centrality of Equation (27) which is basic. It is very noticeable that in [9] we have that the following quote is particularly relevant to consider, in lieu of our results

"Thus, if advanced projects on the detection of GWs will improve their sensitivity allowing to perform a GWs astronomy (this is due because signals from GWs are quite weak) [1], one will only have to look the interferometer response functions to understand if General Relativity is the definitive theory of gravity. In fact, if only the two response functions (2) and (19) will be present, we will conclude that General Relativity is definitive. If the response function (22) will be present too, we will conclude that massless ScalarTensor Gravity is the correct theory of gravitation. Finally, if a longitudinal response function will be 
present, i.e. Equation (25) for a wave propagating parallel to one interferometer arm, or its generalization to angular dependences, we will learn that the correct theory of gravity will be massive Scalar-Tensor Gravity which is equivalent to $f(R)$ theories. In any case, such response functions will represent the definitive test for General Relativity. This is because General Relativity is the only gravity theory which admits only the two response functions (2) and (19) [2] [6]. Such response functions correspond to the two "canonical" polarizations $h+$ and $h \times$. Thus, if a third polarization will be present, a third response function will be detected by GWs interferometers and this fact will rule out General Relativity like the definitive theory of gravity".

We argue that a third polarization in Gravitational waves from the early universe may be detected, if there is proof positive that in the pre Planckian regime that the Corda conjecture [9] as given below, namely if the following analysis is part of our take on relic gravitational waves, is supported by the kinetic energy being larger than the potential energy, namely what if

"The case of massless Scalar-Tensor Gravity has been discussed in [2] [10] with a 'bouncing photons analysis' similar to the previous one. In this case, the line-element in the TT gauge can be extended with one more polarization, labelled with $\Phi(t+z)$, i.e. ..."

This ends our recap of the section given in [8] which we think is important.

What we are arguing for is that the choice of the vacuum energy as given by Equation (27) may give conclusive proof as to satisfy the Corda conjecture and his supposition as to the existence of an additional polarization [9]. We will, in the future try to extend our results so as to determine if Equation (27) either falsifies or supports the existence of a $3^{\text {rd }}$ polarization. Which will be a way to determine the final disposition of GR as THE theory of Cosmology, or open up the possibility of alternate theories. It is an issue which we think will require extreme diligence. While ending our query as to the possible existence of a third polarization we should mention what would be the supreme benefit of our upcoming analysis of Equation (27), namely how to avoid the conflating of dust, with gravitational waves, i.e. the tragic Bicep 2 mistake [10]-[13].

\section{How to Avoid the Bicep 2 Fiasco}

The main agenda would be in utilization of Equation (27) to help nail down a range of admissible frequencies which would be to avoid [10]-[13] conflating the frequencies of collected Gravitational wave signals from relic cosmological conditions (or would be signals) with those connected with Dust generated Gravitational wave signals, especially from dust conflated with Galaxy formation in the early universe. More than anything else, we need to find, likely narrow (?). Frequency ranges, which would be commensurate with Equation (27), and to use advanced detector technology. Of course such a search would be hard. But it also would be a way, with due diligence as to answer questions raised by the Author in [14]. In doing so, the relative flatness of the early universe and its departure from curved space conditions would be a great way to answer the suppositions raised in [8] [9] as well.

\section{Conclusions}

The previously done work by the author as to graviton production invoking nonlinear electrodynamics in cosmology was re-introduced for the purpose as to density functions which are used to create an upper bound to the largest initial time step, in cosmological evolution. Counter intuitively, Equation (22) has no connection as to the scaled value of a vacuum energy as given in Equation (23), which suggests that when frequency rises, as may be connected to alternative values of cosmology, that different processes as to graviton production, as exemplified by Equation (25) still keep a sharp independence as to initial time size as we state it for Equation (22). Keep in mind, that what is being attempted is to upgrade work represented by Maggiore [5], in what may become gravitational astronomy, once falsifiable experimental procedures are agreed upon for vetting a minimal time step. In a future article, the author intends to represent the formation of a minimum time step as a classical back drop for the formation of quantum gravity, i.e. the minimum time step will be a pre cursor to the development of quantum gravity (formation of massive gravitons).

Equation (27) and Equation (28) in themselves argue for an NLED influenced cosmological "vacuum energy", i.e. what we observe, initially is that the above, using $a\left(t_{\text {initial }}\right) \sim 10^{-30}$ and Equation (28) that the value of $\Lambda$ 
would be greater than the present value of the cosmological constant, perhaps by $10^{10}-10^{20}$, arguing that some form of quintessence is argued for. But this value of Equation (27) is far lower than the $10^{120}$ overshoot, obtained by traditional QFT methods [7]. It neatly allows for an investigation as to [14] flatness issues as well as gives credence to what is raised in [8] [9] as far as the existence, of an additional GW polarization.

We wish to point to an earlier paper by Beckwith, and Glinka [15] which has some of the same formalism, but which is geared toward proving inputs from a multiverse. The document so tendered now, has little of the same orientation, plus the emphasis in this paper is to vacuum energy due to conditions in the present universe, without speculation as to multiverses. In addition, [15] makes claims as to conflating gravitons with dark energy. Such speculation is beyond the scope of the document which has been submitted and which has to be proven, experimentally.

We wish to confirm, experimentally, what has been brought up as to vacuum energy, and the critical detail of stating, as done in our document that $\Lambda$ would be greater than the present value of the cosmological constant, perhaps by $10^{10}-10^{20}$, arguing that some form of quintessence is argued for. After this is confirmed, speculations as to conflating gravitons, directly with dark energy may be investigated experimentally. Sadly, the author sees little hope of confirming if the multiverse construction of [15] has any chance of being experimentally tested, in the foreseeable future. Since our orientation, in this paper is the documentation of conditions which may be accessible to experimental vetting, we have to delay for the time being the interesting multiverse speculations of [15] as something which may be tested in the middle of this century, but not now.

\section{Acknowledgements}

This work is supported in part by National Nature Science Foundation of China grant No. 11375279.

\section{References}

[1] Berry, M.V. (1976) Principles of Cosmology. Cambridge University Press, Cambridge, UK.

[2] Camara, C.S., de Garcia Maia, M.R., Carvalho, J.C. and Lima, J.A.S. (2004) Nonsingular FRW Cosmology and Non Linear Dynamics. Physical Review D: Particles and Fields, 69, Article ID: 063501.

[3] Zeld'dovich, Y.B. (1972) The Creation of Particles and Antiparticles in Electric and Gravitational Fields. In: Klauder, J., Ed., Magic without Magic, John Archibald Wheeler, W. H. Freeman and Company, San Francisco, 277-288.

[4] Dupays, A., Rizzo, C., Bakalov, D. and Bignami, G.F. (2008) Quantum Vacuum Friction in Highly Magnetized Neutron Stars. Europhys.Lett., 82, 69002.

[5] Maggiorie, M. (2008) Gravitational Waves, Volume 1, Theory and Experiments. Oxford University Press, Oxford, UK.

[6] Garattini, R. (2006) Effective Action, Massive Gravitons and the Cosmological Constant. Journal of Physics: Conference Series, 33, 215-220. http://iopscience.iop.org/1742-6596/33/1/021/pdf/1742-6596_33_1_021.pdf

[7] Ibanez, L.E. and Urganga, A.M. (2012) String Theory and Particle Physics, An Introduction to String Phenomenology. Cambridge University Press, Cambridge, UK.

[8] Beckwith, A.W. (in press) Gedanken Experiment Examining How Kinetic Energy Would Dominate Potential Energy, in Pre Planckian Space-Time Physics, and Allow Us to Avoid the BICEP 2 Mistake. To be published in JHEPGC, in 2016.

[9] Corda, C. (2009) Interferometric Detection of Gravitational Waves: The Definitive Test for General Relativity. International Journal of Modern Physics D, 18, 2275-2282. http://arxiv.org/abs/0905.2502 http://dx.doi.org/10.1142/S0218271809015904

[10] Van Den Broeck, C. (2015) Gravitational Wave Searches with Advanced LIGO and Advanced Virgo. http://arxiv.org/pdf/1505.04621v1.pdf

[11] Das, S., Mukherje, S. and Souradeep, T. (2015) Revised Cosmological Parameters after BICEP 2 and BOSS. JCAP, 2, 016. http://arxiv.org/abs/1406.0857

[12] Cowen, R. (2015) Gravitational Waves Discovery Now Officially Dead; Combined Data from South Pole Experiment BICEP2 and Planck Probe Point to Galactic Dust as Confounding Signal. http://www.nature.com/news/gravitational-waves-discovery-now-officially-dead-1.16830

[13] Cowen, R. (2014) Full-Galaxy Dust Map Muddles Search for Gravitational Waves. http://www.nature.com/news/full-galaxy-dust-map-muddles-search-for-gravitational-waves-1.15975

[14] Beckwith, A. (2015) Geddanken Experiment for Degree of Flatness, or Lack of, in Early Universe Conditions. Ac- 
cepted for publication, JHEPGC. http://vixra.org/abs/1510.0108

[15] Beckwth, A. and Glinka, L. (2015) On Axionic Dark Matter, Gravitonic Dark Energy, and Multiverse Cosmology in the Light of Non-Linear Electrodynamics. Astrophysics \& Aerospace Technology Beckwith and Glinka. Journal of Astrophysics \& Aerospace Technology, 3, 112. http://dx.doi.org/10.4172/2329-6542.1000112 